\title{
Cultivar Diversity of Balsam (Impatiens balsamina L.) in Banyumas Regency
}

\author{
Rizki Aulia, Pudji Widodo, Wiwik Herawati \\ Biologi Faculty, Universitas Jenderal Soedirman \\ Jl. dr.Suparno 63 Purwokerto 53122 \\ email : rizkiaulia03@gmail.com
}

\begin{abstract}
Rekam Jejak Artikel:
Diterima : 05/08/2020

Disetujui : 09/12/2020

Abstract

Balsam or garden balsam (Impatiens balsamina L.) is a widely grown flowering plant belonging to the family Balsaminaceae. The most conspicuous part to distinguish the balsam is the difference in the flower shape and colors of each cultivar. The purpose of this research is to find out the cultivars diversity of the balsam. The method used in this study was survey with purposive sampling. The variables observed in this study was morphological characteristics including the stem, leaves, flowers, fruits, and seeds. The data obtained were analysed descriptively. The result of this study showed that there were 15 cultivars of I. Balsamina i.e. 'Pinkish White 5 Petal', 'Mix Pink Camellia, 'Pinkish White Camellia, 'Vivid Pink', 'White', 'Light Pink', 'Pinkish White', 'Light Magenta', 'Vivid Red', 'Red Camellia, 'Reddish Camellia, 'Rose Red Camellia, 'Vivid Magenta Camellia, 'Rose Green Camellia, and 'Vivid Pink Camellia'.

Key words: balsam, Balsaminaceae, cultivar, diversity, systematics
\end{abstract}

\section{INTRODUCTION}

The balsam (Impatiens balsamina L.) is an annual herb with $30-80 \mathrm{~cm}$ tall (Backer \& Brink, 1963). The balsam flowers color are pink, red, mauve, lilac, or white. The balsams have a short life cycle, large flowers, and a rather precise differentiation of color classes (Meenu et al., 2015). The flower is very attractive for research, its flowering process requires short-day conditions, and flower reversion can be obtained expectedly after conversion to long-day conditions (Pouteau et al., 1998). One of the best-known characteristics of Impatiens is the explosive dehiscence of the fivevalved, loculicidal capsule. The ripe seed capsules undergo explosive dehiscence (Meenu et al., 2015). The valves of the fruit roll-up inwardly and acropetally, which causes the seeds to be dispersed in all directions. What remains is the central axis of the fruit on which the seeds were attached, and at the top, the spirally winded valves (Caris et al., 2006).

Impatiens balsamina or balsam or garden balsam a species of Impatiens native to southern Asia in India(Rejimon and Varghese, 2015), it belongs to the family of Balsaminaceae. Synonyms of $I$. balsamina is I. coccinea, I. corneta, and Balsamina hortensis. The family consist of more than 1,000 species(Janssens et al., 2009), but only two genera are recognized (Meenu et al., 2015), the large genera Impatiens L. and Hydrocera Blume (Caris et al., 2006). The genera of Impatiens is found in almost all regions in Indonesia such as Java, Sumatra, Kalimantan, Sulawesi, and Papua. Many among them are endemic types. In Indonesia found \pm 60 types of Impatiens with highest diversity is in Sumatra \pm 40 types (Utami, 2012).

The balsams has short life cycle, large flowers, and rather precise differentiation of color classes. The flowers color are pink, red, mauve,lilac, or white (Meenu et al., 2015). According to Tanem \& Williamson (2002) there are several double-flowered I. balsamina cultivars such as 'Camellia Flowered', 'Tom Thumb', and 'Topknot'. Newcomb (1985) also reported that there are several cultivars of garden balsam, namely 'Camellia Flowered', 'Carnation 
Flowered', Double Dwarf Rose Flowered', 'Isabella', 'Rose Flowered', 'Solferino', 'Maiden Blush', 'White Perfection', 'Kermesina', 'Pink Perfection', 'Queen', 'Scarlet Splendens', 'Sunshine', and 'Victoria'. Pal (2015) reported that two strains of annual doubleflowered balsam cultivar,'Tall Double Mix', 'Rose Flowered', 'Royal Balsam Mix', and 'Camellia Flowered'

Balsams are found growing naturally from 0 $\mathrm{m}$ to $1250 \mathrm{~m}$ asl in wetlands, rather open places or as forest undergrowth. In cultivation this plant thrives in soil that is rich in nutrients and loose with water that is easily reached. In the tropics it usually blooms throughout the year (PROSEA, 1999). The garden balsam contains anthocyanin compounds, which have antioxidant and antibacterial.

The sampling areas include Purwokerto areas, Kedung Banteng, Baturraden \& Sumbang at an altitude of between $\pm 25-1000 \mathrm{~m}$ asl where the Purwokerto region is located at an altitude of 25-100 masl and some areas of Kedung Banteng, Baturraden, and Sumbang are located at an altitude of 500-1000 masl (Banyumas Regency Goverment, 2018). Based on the annual rainfall class, the Banyumas Regency area is included in the wetland category (Ministri of Agricultur, 2015). Based on the type of climate according to (Schmidt \& Ferguson, 1951) shows that the Banyumas Regency has a very wet climate. Average annual temperatures range from $26.1{ }^{\circ} \mathrm{C}-28$ ${ }^{\circ} \mathrm{C}$ with a minimum temperature of $21.2{ }^{\circ} \mathrm{C}$ and a maximum temperature of $32.8{ }^{\circ} \mathrm{C}$. The condition of the environment is very suitable with the conditions of garden balsam growing, but at this time not yet known how the diversity of garden balsam in Banyumas District, therefore its really need to do this research.

\section{MATERIALS AND METHODS}

The materials used in this research were 15 cultivars of balsam (Impatiens balsamina L.) and alcohol $70 \%$. This research was conducted in
Banyumas Regency with coordinate S $7^{\circ} 15^{\prime} 05^{\prime \prime}$ 7³7'10" E109 11'17"-109 27'15" which is consist of seven sub-district namely North Purwokerto, East Purwokerto, West Purwokerto, South Purwokerto, Kedung Banteng, Baturraden, and Sumbang. In each sub-district, three villages were sampled, namely North Purwokerto (Grendeng, Sumampir, and Pabuwaran), East Purwokerto (Arcawinangun, Mersi, and Purwokerto Wetan), West Purwokerto (Kober, Bantarsoka, and Kedungwuluh), South Purwokerto (Purwokerto Kidul, Tanjung, and Purwokerto Kulon), Kedung Banteng (Beji, Karang Nangka, and Kutaliman), Baturraden (Karangsalam, Karangtengah, and Karangmangu), and Sumbang (Kebanggan, Banteran, and Banjarsari Wetan). The samples were identified by using Backer \& Brink (1963), Newcomb (1985), Pal (2015), and Tanem \& Williamson (2002). The research method used was survey with purposive sampling technique. The variables observed in this study was morphological characteristics including the stem, leaves, flowers, fruits, and seeds. The voucher speciments were stored at Herbarium Fakultas Biologi Unsoed (PUNS) for future references.

\section{RESULTS AND DISCUSSIONS}

This study showed that there were 15 cultivars of balsam (Impatiens balsamina L.) namely 'Pinkish White 5 Petal', 'Mix Pink Camellia, 'Pinkish White Camellia, 'Vivid Pink', 'White', 'Light Pink', 'Pinkish White', 'Light Magenta', 'Vivid Red', 'Red Camellia, 'Reddish Camellia, 'Rose Red Camellia, 'Vivid Magenta Camellia, 'Rose Green Camellia, and 'Vivid Pink Camellia'.

Impatiens balsamina 'Reddish Camellia' is the most numerous found cultivar because in twenty one villages was found 29 individuals while I. balsamina 'Rose Red Camellia', I. balsamina 'Vivid Magenta Camellia', and I. balsamina 'Pinkish White 5 Petal' are the most fewest found cultivar because only 1 individu were found from twenty-one villages in 
BioEksakta: Jurnal Ilmiah Biologi Unsoed

Volume 2, Nomor 3 (2020): 418 - 426

seven sub-district. Based on the survey of garden balsam, commonly people called this plant with tanaman kingkong, bunga kingkong or mihong. The garden balsam mostly cultivated by people in their house but also around the civil settlement. The cultivation of garden balsam mostly easy because the fruit capsule will explodes and releasing the seeds in random direction also people only spread out the seed randomly.

\section{Key to The Species}

1. a. Stem purely green..... .2

b. Stem contain red color

2. a. Petal number 5 1. I. balsamina 'Pinkish White 5 Petal'

b. Petal number not 5

.3

3. a. Petal number 10

b. Petal number 3 .

4. a. Leaf width $2-3 \mathrm{~cm}$

b. Leaf width $2.5-4 \mathrm{~cm}$

2.I. balsamina 'Mix Pink Camellia'

5. a. Plant height more than $1 \mathrm{~m}$ tall 4. I. balsamina'Vivid Pink'

b. Plant height less than $1 \mathrm{~m}$ tall.

5. I. balsamina'White'

6. a. Petal color white; fruit length up to $2.2 \mathrm{~cm}$ '

b. Petal color light pink; fruit length less than $1.8 \mathrm{~cm}$

7. a. Fruit stalk color reddish

b. Fruit stalk color green. 3. I. balsamina 'Pinkish White Camellia'

a. Fruit length up to $3 \mathrm{~cm}$. 6. I. balsamina'Light Pink'

b. Fruit length up to $2.5 \mathrm{~cm}$. 7. I. balsamina'Pinkish White'

9. a. Spur color whitish

b. Spur color not whitish

10.a. Petiole color green....

b. Petiole color reddish.

11a. Leaf blade linier.

b. Leaf blade oblong-lanceolate

12a. Stem base diameter up to.

b. Stem base diameter up to $2 \mathrm{~cm}$

8. I. balsamina'Rose Red Camellia'

3a. Petiole length up to $2 \mathrm{~cm}$; corolla color vivid magenta

b. Petiole length less than $2 \mathrm{~cm}$; corolla color red.

14a. Leaf length up to $8.5 \mathrm{~cm}$; stem color red.

9. I. balsamina'Vivid Red'

b. Leaf length up to $12.5 \mathrm{~cm}$; stem color reddish green

10. I. balsamina 'Light Magenta' 11

11. I. balsamina'Rose Green Camellia'

12. I. balsamina'Vivid Pink Camellia'

12 13
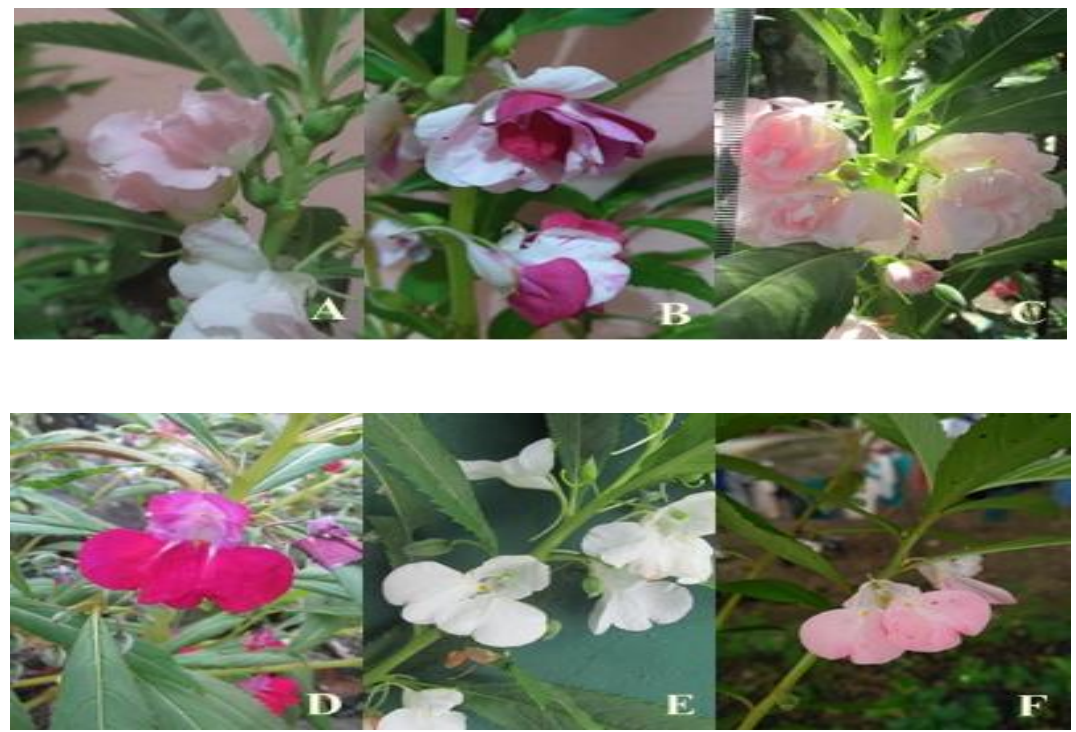

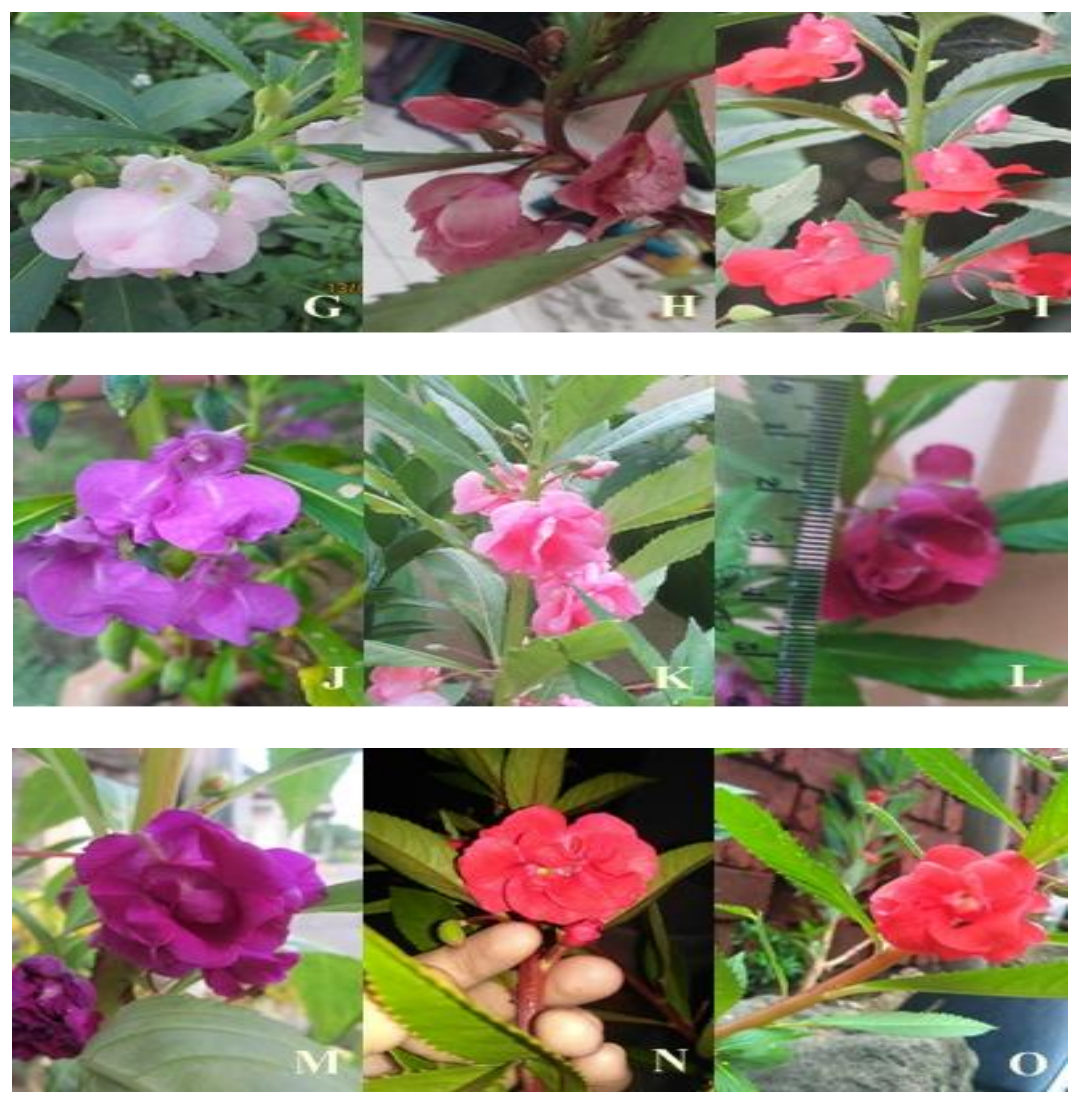

Figure 2. Cultivars of Balsam (Impatiens balsamina L. ). A. 'Pinkish White 5 Petal', B. 'Mix Pink Camellia', C. 'Pinkish White Camellia ', D. 'Vivid Pink', E. 'White', F. 'Light Pink', G. 'Pinkish White', H. 'Rose Red Camellia', I. 'Vivid Red', J. 'Light Magenta', K. 'Rose Green Camellia', L. 'Vivid Pink Camellia', M. 'Vivid Magenta Camellia', N. 'Reddish Camellia', O. 'Red Camellia'.

The description of each cultivars are as follows :

\section{Impatiens balsamina L. 'Pinkish White 5 Petal'}

Annual herb; stem erect and branched, 50-60 $\mathrm{cm}$ tall, 2.5-2.76 cm diameters; light green with swollen nodes; internodes about 0.5-3 cm long; surface shortly hairy. Leaves simple with petiole green; scattered or alternate; oblong-lanceolate; 7-9.5 cm long; 1.5-2.2 cm wide; base cuneate; apex acuminate; margin serrate; leaf surface shortly hairy; upper surface shortly hairy, dark green; lower surface shortly hairy, light green. Flowers simple; lateral or one to three flowers in the leaf axils; two lateral sepals; five petals; spur slender about $1.2-1.8 \mathrm{~cm}$ long; pinkish white. Fruits capsules; stalk green; 1.5$1.8 \mathrm{~cm}$ long; generally striped, eliptical or oval; fivevalved explosive dehiscence; narrowed apex; tomentose or hairy; capsule fleshy; rhegma fruit or ripe capsule explodes releasing seeds by splitting open of locule between the valves called loculicidal capsule; $1.8-2.1 \mathrm{~cm}$ long; $0.52-0.7 \mathrm{~cm}$ in diameters; fruit green. Seeds multiple; ovate; granules; about $0.15-0.28 \mathrm{~cm}$ in diameters; young seed green; old seed dark brown.

Distribution : Mersi, East Purwokerto

\section{Impatiens balsamina L. 'Mix Pink Camellia'}

Annual herb; stem erect and branched, 53-70 $\mathrm{cm}$ tall diameters, $1.18-1.43 \mathrm{~cm}$; light green with swollen nodes; internodes about 0.5-2 cm long; surface shortly hairy. Leaves simple with petiole green; scattered or alternate; oblong-lanceolate; blade 8-13 cm long; 2-3 cm wide; base cuneate; apex acuminate; margin serrate; leaf surface shortly hairy; upper surface shortly hairy, dark green; lower surface shortly hairy, light green. Flowers simple; lateral or one to three flowers in the leaf axils; two lateral sepals; ten petals; spur slender about 2-2.2 cm long; mix light pink, pink, dark pink. Fruits capsule; stalk yellow; 2.1-2.4 cm long; the fruit capsule generally, striped eliptical or oval; five-valved explosive 
dehiscence; narrowed apex; tomentose or hairy; capsule fleshy; rhegma fruit or ripe capsule explodes releasing seeds by splitting open of locule between the valves called loculicid capsule; $1.5-2.5 \mathrm{~cm}$ long; 0.75-0.85 $\mathrm{cm}$ in diameters; fruit green. Seeds multiple; ovate; granules; about $0.16-0.17 \mathrm{~cm}$ in diameters; young seed green; old seed dark brown.

Distribution : Bantarsoka, West Purwokerto \& Karangsalam, Baturraden

\section{Imptiens balsamina L. 'Pinkish White Camellia'}

Annual herb; stem erect and branched; 60-90 $\mathrm{cm}$ tall, 1.32-1.65 cm diameters; stem light green with swollen nodes; internodes about 1-3 cm long; surface shortly hairy. Leaves with petiole green; scattered or alternate; oblong-lanceolate; blade 9-16 cm long; 2.5-4 cm wide; base cuneate; apex acuminate; margin serrate; leaf surface shortly hairy; upper surface shortly hairy, dark green; lower surface shortly hairy, light green. Flowers simple; lateral or one to three flowers located in the leaf axils; two lateral sepals; ten petals; spur slender about $1.7-2 \mathrm{~cm}$ long; pinkish white. Fruits capsules; stalk green; 1.6$2.1 \mathrm{~cm}$ long; the fruit generally striped, eliptical or oval; five-valved explosive dehiscence; narrowed apex; tomentose or hairy; capsule fleshy; rhegma fruit or ripe capsule explodes releasing seeds by splitting open of locule between the valves called loculicid capsule; $2-2.5 \mathrm{~cm}$ long; $0.62-0.80 \mathrm{~cm}$ in diameters; fruit green. Seeds multiple; ovate; granules; about $0.18-0.26 \mathrm{~cm}$ in diameters; young seed green; old seed dark brown.

Distribution : Karangwangkal, North Purwokerto; Karangsalam \& Karangmangu, Baturraden

\section{Impatiens balsamina L. 'Vivid Pink'}

Annual herb; stem erect and branched, 58-145 $\mathrm{cm}$ tall, $1.67-2.21 \mathrm{~cm}$ diameter; light green with swollen nodes; internodes about 1-2 cm long; surface shortly hairy. Leaves simple with petiole green; scattered or alternate; liniar, blade $8-13 \mathrm{~cm}$ long; 1.5 $2 \mathrm{~cm}$ wide; base cuneate; apex acuminate; margin serrate; surface shortly hairy; upper surface shortly hairy, dark green; lower surface shortly hairy, light green. Flowers simple; lateral or one to three flowers in the leaf axils; two lateral sepals; three petals; spur slender about 1.5-1.8 cm long; vivid pink. Fruits capsules; stalk reddish; $1.7-2 \mathrm{~cm}$ long; the fruit capsule generally striped, elliptical or oval; fivevalved explosive dehiscence; narrowed apex; tomentose or hairy; capsule fleshy; rhegma fruit or ripe capsule explodes releasing seeds by splitting open of locule between the valves called loculicidal capsule; 1.5-2 cm long; 0.77-0.97 cm in diameters; fruit green. Seeds multiple; ovate; granules; about $0.18-0.19 \mathrm{~cm}$ in diameters; young seed green; old seed dark brown

Distribution : Kober, West Purwokerto; Purwokerto Kidul \& Tanjung, South Purwokerto; Banteran \& Banjarsari Wetan, Sumbang; Karangtengah \& Karangmangu, Baturraden; Beji, Karang Nangka, \& Kutaliman, Kedung Banteng

\section{Impatiens balsamina L. 'White'}

Annual herb; stem erect and branched, 58-70 $\mathrm{cm}$ tall, $1.155-1.20 \mathrm{~cm}$ diameter; light green with swollen nodes; internodes about 1-3 cm long; surface shortly hairy. Leaves simple with petiole green; scattered or alternate; oblong-lanceolate; blade 8.5$11.5 \mathrm{~cm}$ long; 2-2.7 cm wide; base cuneate; apex acuminate; margin serrate; surface shortly hairy; upper surface shortly hairy, dark green; lower surface shortly hairy, light green. Flowers simple; lateral or one to three flowers in the leaf axils; two lateral sepals; three petals; spur slender about 2-3.5 cm long; white. Fruits capsules; stalk green; 1.9-2.2 cm long; the fruit capsule generally striped, eliptical or oval; five-valved explosive dehiscence; narrowed apex; tomentose or hairy; capsule fleshy; rhegma fruit or ripe capsule explodes releasing seeds by splitting open of locule between the valves called loculicidal capsule; $1.5-2.2 \mathrm{~cm}$ long; $0.73-0.96 \mathrm{~cm}$ in diameters; fruit green. Seeds multiple; ovate; granules; about 
0.20-0.27 cm in diameters; young seed green; old seed dark brown.

Distribution : Sumampir, North Purwokerto; Mersi, East Purwokerto; Kober, West Purwokerto; Karangsalam, Baturraden; Beji \& Kutaliman, Kedung Banteng

\section{Impatiens balsamina L. 'Light Pink'}

Annual herb; stem erect and branched, 60-70 cm tall; 50-1.65 cm diameter; light green with swollen nodes; internodes about 1-2 cm long; surface shortly hairy. Leaves simple with petiole green; scattered or alternate; oblong-lanceolate; blade 8-12.7 cm long; 1.7-3 cm wide; base cuneate; apex acuminate; margin serrate; surface shortly hairy; upper surface shortly hairy, dark green; lower surface shortly hairy, light green. Flowers simple; lateral or one to three flowers in the leaf axils; two lateral sepals; three petals; spur slender about $1.2-1.4 \mathrm{~cm}$ long; light pink. Fruits capsules; stalk reddish; 2-2.5 cm long; the fruit capsule generally striped, eliptical or oval; five-valved explosive dehiscence; narrowed apex; tomentose or hairy; capsule fleshy; rhegma fruit or ripe capsule explodes releasing seeds by splitting open of locule between the valves called loculicidal capsule; $1.1-1.7 \mathrm{~cm}$ long; $0.94-1 \mathrm{~cm}$ in diameters; fruit green. Seeds multiple; ovate; granules; about $0.19-0.22 \mathrm{~cm}$ in diameters; young seed green; old seed dark brown.

Distribution : Banteran \& Banjarsari Wetan, Sumbang; Karangtengah \& Karangmangu, Baturraden; Beji \& Kutaliman, Kedung Banteng

\section{Impatiens balsamina L. 'Pinkish White'}

Annual herb; stem erect and branched;, 55-70 $\mathrm{cm}$ tall; $1.05-1.35 \mathrm{~cm}$ diameters; light green with swollen nodes; internodes about 1-2 cm long; surface shortly hairy. Leaves simple with petiole green; scattered or alternate; oblong-lanceolate; blade 8-16.5 cm long; 1.8-3 cm wide; base cuneate; apex acuminate; margin serrate; surface shortly hairy; upper surface shortly hairy, dark green; lower surface shortly hairy, light green. Flowers simple; lateral or one to three flowers in the leaf axils; two lateral sepals; three petals; spur slender about 1.8-2 cm long; pinkish white. Fruits capsules; stalk green; $1.5-1.8 \mathrm{~cm}$ long; the fruit capsule generally striped, eliptical or oval; five-valved explosive dehiscence; narrowed apex; tomentose or hairy; capsule fleshy; rhegma fruit or ripe capsule explodes releasing seeds by splitting open of locule between the valves called loculicidal capsule; $1-1.2 \mathrm{~cm}$ long; $0.72-0.77 \mathrm{~cm}$ in diameters; fruit green. Seeds multiple; ovate; granules; about 0.19-0.22 cm in diameters; young seed green; old seed dark brown.

Distribution : Kober, West Purwokerto; Karangsalam, Baturraden

\section{Impatiens balsamina L.. 'Rose Red Camellia'}

Annual herb; stem erect and branched, 75-85 $\mathrm{cm}$ tall, 1.46-2.12 cm diameters; stem red with swollen nodes; internodes about 1-2.5 cm long; surface shortly hairy. Leaves simple with petiole red; scattered or alternate; oblong-lanceolate; blade 10-15 cm long; 2.5-4 cm wide; base cuneate; apex acuminate; margin serrate; surface shortly hairy; upper surface shortly hairy, dark green; lower surface shortly hairy, light green. Flowers simple; lateraly or one to three flowers located in the leaf axils; two lateral sepals; ten petals; spur slender about 2.3-2.5 cm long; very light rose. Fruits capsules; stalk reddish; 2-2.3 cm long; the fruit capsule generally striped, eliptical or oval; five-valved explosive dehiscence; narrowed apex; tomentose or hairy; capsule fleshy; rhegma fruit or ripe capsule explodes releasing seeds by splitting open of locule between the valves called loculicid capsule; $2.5-3 \mathrm{~cm}$ long; 0.75-0.96 cm in diameters; fruit green. Seeds multiple; ovate; granules; about $0.20-0.24 \mathrm{~cm}$ in diameters; young seed green; old seed dark brown.

Distribution: Karangsalam, Baturraden 


\section{Impatiens balsamina L. 'Vivid Red'}

Annual herb; stem erect and branched, 68-70 $\mathrm{cm}$ tall, 1.94-2.05 cm diameters; reddish-green with swollen nodes; internodes about 0.5-3 cm long; surface shortly hairy. Leaves simple with petiole green; scattered or alternate; oblong-lanceolate; blade 8-11 cm long; 2-2.5 cm wide; base cuneate; apex acuminate; margin serrate; surface shortly hairy; upper surface shortly hairy, dark green; lower surface shortly hairy, light green. Flowers simple; lateral or one to three flowers in the leaf axils; two lateral sepals; three petals; spur slender about $1.5-1.8 \mathrm{~cm}$ long; vivid red. Fruits capsules; stalk reddish; 2.4-2.5 $\mathrm{cm}$ long; the fruit capsule generally striped, eliptical or oval; five-valved explosive dehiscence; narrowed apex; tomentose or hairy; capsule fleshy; rhegma fruit or ripe capsule explodes releasing seeds by splitting open of locule between the valves called loculicid capsule; $1.6-2.2 \mathrm{~cm}$ long; $0.85-1.19 \mathrm{~cm}$ in diameters; fruit green. Seeds multiple; ovate; granules; about 0.19-0.24 cm in diameters; young seed green; old seed dark brown.

Distribution : Mersi, East Purwokerto; Banteran \& Banjarsari Wetan, Sumbang; Karangtengah, Baturraden

\section{Impatiens balsamina L. 'Light Magenta'}

Annual herb; stem erect and branched, 42-90 $\mathrm{cm}$ tall, 2-2.54 cm diameter; reddish-green with swollen nodes; internodes about 0.5-1.5 cm long; surface shortly hairy. Leaves simple with petiole green; scattered or alternate; linear, blade $10-15.5 \mathrm{~cm}$ long; 1.5-2 cm wide; base cuneate; apex acuminate; margin serrate; surface shortly hairy; upper surface shortly hairy, dark green; lower surface shortly hairy, light green. Flowers simple; lateraly or one to three flowers in the leaf axils; two lateral sepals; three petals; spur slender about 1.2-1.4 cm long; light magenta. Fruits capsules; stalk reddish; 2-2.4 cm long; the fruit capsule generally striped, eliptical or oval; five-valved explosive dehiscence; narrowed apex; tomentose or hairy; capsule fleshy; rhegma fruit or ripe capsule explodes releasing seeds by splitting open of locule between the valves called loculicid capsule; $1.6-2 \mathrm{~cm}$ long; $0.60-0.73 \mathrm{~cm}$ in diameters; fruit green. Seeds multiple; ovate; granules; about 0.2-0.25 cm in diameters; young seed green; old seed dark brown.

Distribution : Sumampir, North Purwokerto; Purwokerto Wetan, East Purwokerto; Kober, West Purwokerto; Kebanggan, Banteran, \& Banjarsari Wetan, Sumbang; Karangtengah, Baturraden; Beji \& Karangnangka, Kedung Banteng

\section{Impatiens balsamina L. 'Rose Green Camellia'}

Annual herb; stem erect and branched, 70-80 $\mathrm{cm}$ tall; 1.74-2.80 cm diameter; reddish-green with swollen nodes; surface shortly hairy. Leaves simple with petiole reddish-green; scattered or alternate; liniar; blade 8-13 cm long; 2.3-3.5 cm wide; base cuneate; apex acuminate; margin serrate; surface shortly hairy; upper surface shortly hairy, dark green; lower surface shortly hairy, light green; internodes about $1-5 \mathrm{~cm}$. Flowers simple; lateral or one to three flowers in the leaf axils; two lateral sepals; ten petals; spur slender about $2.5-3.1 \mathrm{~cm}$ long; very light rose. Fruits capsules stalk reddish; $1.5-3 \mathrm{~cm}$ long; the fruit capsule generally striped, eliptical or oval; fivevalved explosive dehiscence; narrowed apex; tomentose or hairy; capsule fleshy; rhegma fruit or ripe capsule explodes releasing seeds by splitting open of locule between the valves called loculicid capsule; $1.4-1.6 \mathrm{~cm}$ long; 0.61-0.64 cm in diameters; fruit green. Seeds multiple; ovate; granules; about 0.14-0.18 cm in diameters; young seed green; old seed dark brown

Distribution : Arcawinangun, East Purwokerto; Kober, West Purwokerto

\section{Impatiens balsamina L. 'Vivid Pink Camellia'}

Annual herb; stem erect and branched, 65-70 $\mathrm{cm}$ tall; 1.10-1.55 cm diameter; reddish-yellow with swollen nodes; internodes about 1.5-2 cm long; 
surface shortly hairy. Leaves simple with petiole reddish; scattered or alternate; oblong-lanceolate; blade 8-11.5 cm long; 1.5-2.5 cm wide; base cuneate; apex acuminate; margin serrate; surface shortly hairy; upper surface shortly hairy, dark green; lower surface shortly hairy, light green. Flowers simple; lateral or one to three flowers in the leaf axils; two lateral sepals; ten petals; spur slender about 1.3-2.2 cm long; vivid pink. Fruits capsules; stalk reddish; 2.1-2.2 cm long; the fruit capsule generally striped, eliptical or oval; five-valved explosive dehiscence; narrowed apex; tomentose or hairy; capsule fleshy; rhegma fruit or ripe capsule explodes releasing seeds by splitting open of locule between the valves called loculicidal capsule; $2-2.5 \mathrm{~cm}$ long; $0.69-1.40 \mathrm{~cm}$ in diameters; fruit green. Seeds multiple; ovate; granules; about $0.17-0.22 \mathrm{~cm}$ in diameters; young seed green; old seed dark brown.

Distribution : Mersi, East Purwokerto; Kebanggan, Sumbang; Karang Nangka, Kedung Banteng

\section{Impatiens balsamina L. 'Vivid Magenta Camellia'}

Annual herb; stem erect and branched, 60-80 $\mathrm{cm}$ tall; 2-2.08 cm diameters; reddish-green with swollen nodes; internodes about 0.5-1.5 cm long; surface shortly hairy. Leaves simple with petiole reddish-green; scattered or alternate; oblonglanceolate; blade 10-14 cm long; 2.5-3.5 cm wide; base cuneate; apex acuminate; margin serrate; surface shortly hairy; upper surface shortly hairy, dark green; lower surface shortly hairy, light green. Flowers simple; lateral or one to three flowers located in the leaf axils; two lateral sepals; ten petals; spur slender about 2.4-2.8 cm long; vivid magenta. Fruits capsules; stalk reddish; 2-3.2 cm long; the fruit capsule generally striped, eliptical or oval; fivevalved explosive dehiscence; narrowed apex; tomentose or hairy; capsule fleshy; rhegma fruit or ripe capsule explodes releasing seeds by splitting open of locule between the valves called loculicidal capsule; $1.5-2.2 \mathrm{~cm}$ long; $0.7-1 \mathrm{~cm}$ in diameters; fruit green. Seeds multiple; ovate; granules; about 0.18 $0.22 \mathrm{~cm}$ in diameters; young seed green; old seed dark brown.

Distribution : Arcawinangun, East Purwokerto

\section{Impatiens balsamina L. 'Red Camellia'}

Annual herb; stem erect and branched, 86-90 $\mathrm{cm}$ tall; 2.33-2.5 cm diameters; stem red with swollen nodes; internodes about $0.5-1.5 \mathrm{~cm}$ long; surface shortly hairy. Leaves simple with petiole red; scattered or alternate; oblong-lanceolate; blade 5.5$8.5 \mathrm{~cm}$ long; 1.6-2 cm wide; base cuneate; apex acuminate; margin serrate; surface shortly hairy; upper surface shortly hairy, dark green; lower surface shortly hairy, light green. Flowers simple; lateral or one to three flowers in the leaf axils; two lateral sepals; ten petals; spur slender about 0.8-3.3 cm long; red. Fruits capsules; stalk reddish; 2.6-2.8 cm long; the fruit capsule generally striped, eliptical or oval; five-valved explosive dehiscence; narrowed apex; tomentose or hairy; capsule fleshy; rhegma fruit or ripe capsule explodes releasing seeds by splitting open of locule between the valves called loculicid capsule; $1.5-2 \mathrm{~cm}$ long; $0.73-1 \mathrm{~cm}$ in diameters; fruit green. Seeds multiple; ovate; granules; about 0.14-0.2 $\mathrm{cm}$ in diameters; young seed green; old seed dark brown.

Distribution : Arcawinangun \& Mersi, East Purwokerto; Bantarsoka; West Purwokerto; Purwokerto Kulon, South Purwokerto; Kebanggan \& Banteran, Sumbang; Karangtengah, Baturraden; Beji \& Karang Nangka, Kedung Banteng

\section{Impatiens balsamina L. 'Reddish Camellia'}

Annual herb; stem erect and branched, 72-86 $\mathrm{cm}$ tall; 1.53-2.23 cm diameter; reddish-green with swollen nodes; internodes about 1.5-2 cm long; surface shortly hairy Leaves simple with petiole reddish-green; scattered or alternate; oblonglanceolate; blade 6-12.5 cm long; 2-3 cm wide; base cuneate; apex acuminate; margin serrate; surface shortly hairy; upper surface shortly hairy, dark green; 
lower surface shortly hairy, light green. Flowers simple; lateral or one to three flowers in the leaf axils; two lateral sepals; ten petals; spur slender about 1.7-1.9 cm long; red. Fruits capsules; stalk reddish; 1.9-2.1 cm long; the fruit capsule generally striped, eliptical or oval; five-valved explosive dehiscence; narrowed apex; tomentose or hairy; capsule fleshy; rhegma fruit or ripe capsule explodes releasing seeds by splitting open of locule between the valves called loculicidal capsule; $1.8-2 \mathrm{~cm}$ long; $0.75-1.1 \mathrm{~cm}$ in diameters; fruit green. Seeds multiple; ovate; granules; about $0.15-0.22 \mathrm{~cm}$ in diameters; young seed green; old seed dark brown.

Distribution: North Purwokerto; West Purwokerto; Purwokerto Kidul \& Purwokerto Kulon, South Purwokerto; Kebanggan \& Banteran, Sumbang; Karangtengah \& Karangmangu, Baturraden; Karang Nangka \& Kutaliman, Kedung Banteng

\section{CONCLUSION}

There were 15 cultivars of I. balsamina found I. balsamina. Cultivar 'Reddish Camellia' is the most numerous cultivar because found 29 individuals while 'Rose Red Camellia', 'Vivid Magenta Camellia', and 'Pinkish White 5 Petal' were the most fewest found cultivar because only one individu.

\section{REFERENCES}

Backer, C. A. \& Brink, R. C. B. v. d., 1963. Flora of Java (Spermatophytes Only). Groningen: P. Noordhoff.

Caris, P. L., Geuten, K. P., Janssens, S. B. \& Smets, E. F., 2006. Floral Development in Three Species of Impatiens (Balsaminaceae). American Journal of Botany, 93(1) : 1-14.

KPU Kabupaten Banyumas, 2019. Peta Banyumas KPU Banyumas. [Online] Available at: http://kab-banyumas.kpu.go.id/ setelah-penetapan-dapil-kpuajak-parpolbelajar-tata-cara-penghitungan-kursi/petabanyumas-kpubanyumas/ [Diakses 27 August 2019].

Kementrian Pertanian, 2015. Peta Pengembangan Kawasan Padi dan Kedelai Kabupaten Banyumas, Provinsi Jawa Tengah, Jakarta: Sekretariat Jenderal Kementrian Pertanian.
Meenu, B., D., N. E., Rejimon, G. \& Varghese, A., 2015. Impatiens balsamina : An overview. Journal of Chemical and Pharmaceutical Research, 7(9) : 16-21.

Newcomb, P. C., 1985. Popular Annuals of Eastern North America. Washington, D.C.: Dumbartone Oaks.

Pal, S., 2015. Evaluation and Genetic Analysis in Various Genotypes of Balsam (Impatiens balsamina L.). Thesis : 1-147.

PROSEA Indonesia, 1999. Sumber Daya Nabati Asia Tenggara. Dalam: R. Lemmens \& N. W. Soetjipto,. Tumbuh-Tumbuhan Penghasil Pewarna dan Tanin. 3. Bogor: PT. Balai Pustaka, 93.

Pemerintah Kab. Banyumas, 2018. Ketinggian Wilayah Kabupaten Banyumas dari Permukaan Laut. [Online]Available at: http://data.jatengprov.go.id/dataset/ketinggianwilayahkabupaten banyumas-dari-permukaanlaut[Diakses 27 August 2019].

Pouteau, S., Tooke, F. \& Battey, N., 1998. Quantitative Control of Inflorescence Formation in Impatiens balsamina. Plant Physiol, Volume 118 : 1191-1201.

Schmidt, F. H. \& Ferguson, J. H., 1951. Rainfall Types based on Wet and Dry period Ratios for indonesia with Western New Guinea.. 42. Jakarta: Djawatan meteorologi dan Geofisik.

Tanem, B. \& Williamson, D., 2002. Annuals fo Northern California. Canada: Lone Pine Publishing.

Utami, N., 2012. Impatiens talakmauensis (Balsaminaceae), A New Species from Western Sumatra, Indonesia. Acta Phytotaxonomica Geobotanica, 63(1) : 51-5

D, N. E., Rejimon, G. and Varghese, A. 2015. Impatiens balsamina: An overview, Journal of Chemical and Pharmaceutical Research.

Pharmacon. 2017. Formulasi Sediaan Sabun Cair Antiseptik Ekstrak Etanol Bunga Pacar Air (Impatiens balsamina L.) dan Uji Efektivitasnya Terhadap Bakteri Staphylococcus Aureus Secara In Vitro'. Pharmacon. doi: 10.35799/pha.6.2017.16885.

Janssens, S. B. et al. (2009) 'Rapid radiation of Impatiens (Balsaminaceae) during Pliocene and Pleistocene: Result of a global climate change', Molecular Phylogenetics and Evolution. doi: 10.1016/j.ympev.2009.04.013. 